\title{
1 Diagnostic imaging techniques of the respiratory tract of sheep
}

3 Castells, E. 1; Lacasta, D. ${ }^{2}$; Climent, M. 3; Pérez, M.1 ; Sanromán, F.1; Jimenez, C. 2;

4 Ferrer, L.M. 2

$5 \quad{ }^{I}$ Centro Clínico Veterinario de Zaragoza. Spain.

$6 \quad{ }^{2}$ Animal Pathology Department. Veterinary Faculty of Zaragoza. Spain.

$7 \quad{ }^{3}$ Anatomy, embryology and animal genetics Department. Veterinary Faculty of Zaragoza. Spain.

8 Corresponding autor: 1mferrer@unizar.es

\section{ABSTRACT}

11 Diagnostic imaging techniques are very useful non-invasive methods to obtain medical images for the 12 diagnosis of respiratory diseases in sheep.

13 The use of ultrasound and thermographic cameras must be enhanced at farm level with the objective of 14 assisting in the diagnosis of major respiratory diseases present in sheep farms. X-ray and, particularly, 15 computed tomography are very interesting tools to facilitate the understanding of the main pathological 16 processes in sheep, especially at the respiratory level.

17 This article shows more than 40 images of thermograms, X-ray, ultrasonography and computed tomography 18 of the most significant respiratory diseases in sheep.

19 Keywords: sheep, respiratory tract, diagnostic imaging techniques, thermography, ultrasonography, X-ray and computed tomography

\section{Introduction}

Diagnostic imaging techniques are non-invasive methods to obtain medical images for the diagnosis of diseases. Diagnostic imaging also establishes a database of normal anatomy and physiology as a reference to identify abnormalities in the different species.

For the diagnosis of respiratory diseases, the most useful diagnostic imaging techniques are ultrasonography, radiography, infrared thermography and computed tomography. Although it is obvious that some of these techniques will not be applicable in field conditions, these have been introduced in this article for scientific purposes and to provide a better understanding and comprehension of some respiratory pathologies.

Ultrasonographic examination has been widely used in the diagnosis of pregnancy in sheep; however it does not play still a major role in general clinical practice for the diagnosis of pathological disorders. Recently, Scott et al. (2017) published a deep review on the use of ultrasonography for the diagnosis of respiratory pathologies in sheep, thus not being this topic the main aim of the present article. Infrared thermography is an innovative non-invasive tool that allows the remote measurement of the surface 
colour thermal image, where each colour corresponds to a specified temperature (Redaelli and

39 Caglio, 2013). These measurements can be evaluated to establish their physiological or 40 pathological meaning, detecting inflammation of the superficial areas and becoming very useful 41 to identify subclinical signs before the disease progresses (Luzi et al., 2013). These cameras will 42 be very valuable in the diagnosis of upper respiratory tract pathologies in sheep.

$\mathrm{X}$-ray imaging is based on the absorption of X-rays as they pass through a patient's body.

Depending on the amount absorbed in a particular tissue, a different amount of X-rays will pass through the body and interact with the detection device (X-ray film or other image receptor) to finally provide a 2-dimensional projection image of the tissues within the patient's body (radiography). Nowadays, this technique is the most commonly used for the diagnosis of respiratory diseases in small animals, yet in sheep production it has not a widespread use.

Finally, computed tomography, also known as CT scanner, is also based on the variable absorption of X-rays by different tissues. However, CT provides a different form of imaging known as cross-sectional imaging. The origin of the word "tomography" is from the Greek word "tomos" meaning "slice" or "section" and "graphe" meaning "drawing". Therefore, this system provides images that are slices of the anatomy of the animal. For the moment, and due to the high price of these devices, CT is only used with research proposes in sheep, being very valuable to understand the different respiratory pathologies. In this article different CT images of the main respiratory pathologies in sheep are shown.

In order to improve the understanding of respiratory diseases and their diagnosis, the text has been divided into two parts: upper respiratory tract pathologies, those affecting nasal cavity and paranasal sinuses, nasopharynx and larynx, and lower respiratory tract pathologies affecting trachea, lung and pleura.

The devices used to perform the images shown in this article are:

Thermographic camera: FLIR E63900, T198547. Images were performed at the Ruminant Clinical Service of the Veterinary Faculty of Zaragoza, Spain.

Portable ultrasound machine: VET EICKCMEYER Magic 5000 3.5-5 MHz. Images were performed at the Ruminant Clinical Service of the Veterinary Faculty of Zaragoza, Spain.

X-ray equipment: Sedecal, portable generator. SP-vet-4.0 Model. Images were performed at the Equine surgery and medical service of the Veterinary Faculty of Zaragoza, Spain.

Computed axial tomography: General Electric Healthcare. Bivro model of two slides. Images were performed at the Centro Clínico Veterinario of Zaragoza, Spain. 


\section{2. Upper respiratory tract pathologies}

74

75

76

77

78

79

80

81

82

83

84

85

86

87

88

89

90

91

92

93

94

95

96

97

98

99

100

101

102

103

104

105

106

107

\subsection{Head}

\subsubsection{Enzootic nasal adenocarcinoma of sheep}

Enzootic nasal adenocarcinoma (ENA) is a contagious chronic disease of the upper airways in sheep. It has been described in Spanish farms, but also in all over the world (except in New Zeeland and Australia). ENA prevalence in an affected flock is variable, ranging from 0.1$15 \%$ and usually several cases are observed in the same flock. ENA is caused by an oncogenic retrovirus, ENTV-1 (Enzootic nasal tumour virus), that induces neoplastic (adenocarcinoma) growth of the secretory cells from the ethmoidal mucosa (De las Heras et al., 2018). First, a continuous unilateral serous discharge appears to evolve to a bilateral one, causing loss of hair around the nose. The disease is also known as "washed nose disease" (Ferrer et al., 2002). As the tumour grows, the bone around it undergoes pressure and become deformed, being even possible to deform the whole skull in severe cases, provoking exophthalmos and skin fistulation. Furthermore, ENA induces inspiratory dyspnea and snoring sounds. At necropsy, tumour is soft, grey or reddish-white in colour with a fine granular surface and it is covered with clear mucus.

For the diagnosis of this disease, Infrared thermography will be a useful tool. Thermographic camera performs pictures which present a colour scale that covers from cold (green and blue) to hot (yellow, orange, red and white). In the thermal picture (thermogram) of the nostril of a healthy animal, blue and green colours are found (Figs. 1a-1b). This is due to the fact that the air is passing through the nostril refrigerating the area. However, in ENA cases, the thermography shows reddish or even white colours in the posterior segment of the nose, matching the hottest areas (white colour) with the ethmoidal bone, where the ENA is located (Figs. 1c-1d). The nasal cavity presents also a red colour because, due to the obstruction provoked by the tumour, air cannot pass through the nose cooling the area.

In radiography, loss and deformation of the bone tissue in the ethmoidal area can be observed. A grey mass occupies the inside of the nasal chamber, pushing up the nasal bone in a more advanced stage of the disease (Fig. 2a).

Ultrasonography is not a very useful technique for the diagnosis of ENA. Only in the case that the nasal bone is destroyed and becomes a soft tissue, it is possible to see an echogenic tissue in the ethmoidal area with the transducer (Fig. 2b).

The last method described is the computed tomography (CT). This device takes pictures around the whole skull and makes sections or slides of it, being the best method to visualize 
108 tissues comparing to other methods. Therefore, in ENA cases, this method shows the destruction

109 of the ethmoidal bone, the lithic curse of the nasal bone and the soft tissues growing, even before

110 the nasal bone is destroyed and the face deformed (Figs. 2c-2d).

\section{2.1.2. Chronic proliferative rhinitis}

113

114

115

116

Chronic proliferative rhinitis (CPR) in sheep has been associated with Salmonella enterica subsp. diarizonae serotype 61:k:1,5 (7) and causes an inflammation of the ventral nasal turbinates that produces uni-or bilateral thick seromucous nasal discharge, together with wheezing and snoring. These signs persist for several weeks or months, and worsen with almost complete nasal obstruction caused by the presence of proliferating tissue, often visible at the nares. Finally, animals develop severe respiratory distress with a striking mouth breathing (Lacasta et al., 2012). Pathological findings show a swollen ventral turbinates with a roughened surface. The section of the turbinate shows a proliferative tissue that is usually composed of multiple small white or yellow polypoid structures covered by mucus, although only a thickening of the mucosa can be observed (Lacasta et al., 2012; Rubira et al., 2018).

Thermographic images of CPR cases detect high temperatures (white colour) in the nostril area corresponding to the swollen ventral turbinate and a defect in nasal cavity ventilation (Fig. 3a).

In radiography, the affection of the ventral conchae can be seen with two lateral radiographic projections, one of each side. Although this disease does not affect the nasal bone as clearly as the enzootic tumour, an increased opacity is observed inside the ventral nose chamber (Fig. 3b).

Ultrasonography of CPR affected animals is not easy to interpret, mainly when the inflammation of the ventral conchae is not very severe. It is necessary to press the transducer against the nasoincisive notch until the swollen mass makes contact. More echogenic or hyperechoic tissue in the ventral area of the nostril is observed.

Finally, computed tomography enables to obtain a clear image of the damaged tissue and the different stages of development of the disease. It also shows the increase in size of swollen tissues and the bone destruction in more advanced cases (Figs. 3c-3d).

\subsubsection{Oestrosis}

Oestrosis is a common parasitism caused by a fly, Oestrus ovis, and their larval instars. This parasite is widespread in countries where there are a large amount of sheep and goats. Their 
143 presence is associated with hot and dry climates, such as Spain (Gracia et al., 2010), and also

144 countries with extensive and semi-extensive production system flocks (Lucientes et al., 1998).

145 The larvae produce a chronic inflammatory rhinitis, with serous, mucus, purulent or even

146 haemorrhagic discharge from the noses. These secretions are linked to the stage of the larvae and

147 also with the weather, because larvae stop their activity in some weather conditions (winter) and

148 so do the secretions (Alcaide et al., 2003; Ferrer et al., 2002; Gracia et al., 2018). The prevalence

149 of this illness is close to $85 \%$ in some Mediterranean countries.

150 For the diagnosis of this disease, thermal images and ultrasound are not used unless the

151 parasitation is very severe. Radiography offers a poor capacity of detection, because the larvae

152 body is not revealed. Only in some cases, the final stage of larvae (L3) can be seen (Fig. 4a).

153 Computed tomography offers better images. Tomographic pictures show the secretions,

154 the swollen tissues of the turbinates and even the segments of the larvae (Figs. 4b-4c).

\section{2.1.4. Intranasal Abscess}

156

157

158

159

160

161

162

163

164

165

166

167

168

169

170

171

172

173

174

Finally, there are other causes of rhinitis, such as nasal abscesses. These processes are usually caused by bacterial infection and can be formed by pus or by caseous material (Ferrer et al., 2002; Benavides et al., 2015).

For the diagnosis of this pathology, x-ray shows a loss of the bone wall thickness and an increased soft tissue shape where the nasal bone disappears and turbinate bones loss their structure (Fig. 5a).

In thermographic images high temperatures (red and white colours) can be observed on the affected area (Fig. 5b).

Ultrasonography may only be used if the nasal upper side of the bone has suffered a lithic process. In this case, it is possible to put a transducer on the soft injured part and observe an echogenic capsule and underneath hypoechogenic tissues.

7 The CT allows a better view of all the mass and its location within the nasal cavity.

68 Damaged areas and affected tissues can be differentiated as well as injured bone and the loss of 69 its thickness in some places, or even abscess invasion from one to other nasal chamber (Figs. 5c$5 d$ and $6 a-6 b)$.

Pharynx and larynx pathological disorders are not very common in sheep. Only chronic laryngitis associated with laryngeal chondritis has been commonly described in animals of the 
175 Texel breed (Lane et al., 1987). Moreover, it was published a case received in our ruminant

176 clinical service of Laryngeal hemiplegia in a Rasa Aragonesa Ram associated with Sarcocystis

177 infection (Sáez et al., 2003). However, as in other species, processes such as abscesses, foreign

178 bodies or tumours can be located in these areas. Ultrasound and tomographic assessments will

179 allow evaluating the injury and getting a reliable diagnosis.

180 In the Fig. 7a ultrasonography of a ewe with a $7.27 \mathrm{~cm}$ abscess caused by

181 Corynebacterium pseudotuberculosis is revealed. The big abscess pressed the larynx deforming

182 it and causing respiratory distress. Figures $7 \mathrm{~b}, 7 \mathrm{c}$ and $7 \mathrm{~d}$ show an axial and sagittal CT view of

183 the neck of this ewe with the CLA pyogranuloma on the epiglottis pressing the larynx.

\subsubsection{Tracheal crushing}

Tracheal crushing is a common disorder in our area of study. In a survey carried out by our research group, $100 \%$ of analysed farms had animals with lesion in some tracheal rings, 9.95\% out of 7699 examined sheep were affected of tracheal damage. The presence of tracheal injury is clearly influenced by age; $63 \%$ of the affected animals were over 7 years (Ortega et al., 2017). Although the cause of this injury is not well defined yet, it seems to be a disorder associated with the management and the type of feeders during the periods of confinement of the animals.

In this pathology, radiography would be the gold standard diagnosis technique, because it allows assessing the lumen of the trachea and locating the injured area (Fig. 8a), while computed tomography would provide additional details about the process (Fig. 8b).

\section{Lower respiratory tract pathologies}

Ruminants are particularly sensitive animals to the development of lung pathologies, causing relevant economic losses. In lambs, apart from parasitic pneumonias, easy to diagnose with a coprological test, there is basically a pathology affecting the lower respiratory tract, ovine respiratory complex. However, in adults, there are several diseases that settle in the lower tract,

208 the clinical signs, these diseases are not easy to differentiate. Therefore, symptoms of the 
productive processes as gangrenous pneumonia, ovine respiratory complex or even pulmonary

210 adenocarcinoma, can be very similar, becoming imaging diagnosis techniques a very useful tool

211 to reach the final diagnosis.

212 In the study of lower respiratory tract pathologies, only ultrasonography, radiography and

213 computed axial tomography will be shown, because thermography is not a valuable tool in these

214 pathological processes.

\subsection{Maedi-Visna disease}

Maedi-Visna disease is an ovine disease caused by SRLV (small ruminant lentivirus), that induce a systemic infection that may affect in an immunomediated manner an array of target organs, such as lung, central nervous system, mammary gland and joints (Minguijón et al., 2015).

221 The clinical syndrome is caused by interstitial pneumonia that produces severe dyspnea without 222 productive sounds and progressive loss of bodyweight (Luján et al., 2018). However, when the 223 illness is not so advanced, clinical signs can be confusing. Moreover, SRLV infects 224 immunological cells promoting the development of other kind of lung injuries. In a survey carried out in our clinical service, $52.2 \%$ of the animals with interstitial pneumonia related to SRLV presented other lung injury as pleurisy, fibrinous pneumonia, abscesses or gangrenous pneumonia (Lacasta et al., 2016).

Radiography shows, in a more advance stage, a diffused interstitial pattern in all lungs (Fig. 9a). parenchyma (Fig. 9b), although is not easy to differentiate at the initial stages of the disease.

Computed Tomography provides a better detail of the lesion, highlighting the increased $2349 \mathrm{c})$.

\subsection{Ovine Respiratory Complex in adults}

As ovine respiratory complex (ORC) in lambs, in adults, ORC is regarded as a complex 239 disease, involving interaction among host (immunological and physiological), multiple 240 etiological agents (bacteria and mycoplasma) and environmental factors (stressors) and it 241 produces similar lesional pattern (Lacasta et al., 2018). A hyperacute or systemic form, 242 characterized by hemorrhages, and acute and chronic forms, characterized by lung consolidation, 
can be present. Lung consolidation is shown as suppurative (catharral) or fibrinous pneumonia with different degrees of severity.

In a chronic pneumonia related to ORC, radiography shows an alveolar pattern, without an interstitial lesion (Fig. 10a). Opaque alveoli with fluid in bronchial lumen are seen. Ultrasonography displays areas of more echogenic tissue without a clear split of the normal tissue (Fig. 10b). In some cases, it is possible to find abundant purulent foci of different size and texture.

Computed Tomography reveals a better view of the injured areas. Collapsed lung areas are more opaque and whitish (Fig. 10c), while healthy tissue remains the typical grey colour of a lung full of air. It is interesting to highlight that air usually remains inside the thickest bronchia even when they are surrounded by pneumonic tissue. With the software associated to the CT scanner is possible to measure the affected area of the lung. Based on this measurement, the progression of the disease can be followed.

\subsection{Ovine pulmonary adenocarcinoma}

Ovine pulmonary adenocarcinoma (OPA) is a transmissible lung tumour of sheep caused by jaagsiekte sheep retrovirus (JSRV) which induces the transformation of secretory epithelial cells of the distal respiratory tract. Affected animals have dyspnea and moist respiratory sounds, caused by the accumulation of fluid in the respiratory airways. In the final stages of the disease, variable amounts of frothy serous pulmonary fluid (De Las Heras et al., 2003; Cousens et al., 2009) is discharged from the nostrils when the sheep head is lowered ("wheelbarrow" test). At necropsy, neoplastic lesions are diffuse or nodular, grey or purple in colour and have an increased consistency (Ortín et al., 2018).

In the X-ray, a nodular pattern can be observed at the beginning of the process with small and diffused nodules (Fig. 11a). Once tumour nodules converge and form larger lesions, they are more easily detected.

Ultrasonographic examination of ovine pulmonary adenocarcinoma involving the lung surface/visceral pleura reveals hypoechoic areas representing a tumour mass, defined dorsally by a broad hyperechoic line (Scott, 2017). Ultrasound displays echogenic areas that correspond with different-sized neoplastic nodules associated with OPA (Fig. 11b).

Computed tomography provides a clear picture of the tumour nodules inside the lung and their different sizes and locations. This technique also allows serial CTs to evaluate the progression of the disease. At first, it can be observed a nodular pattern with small and diffused dotted that converge in different-sized masses (Fig. 11c). 


\subsection{Verminous pneumonia}

This pneumonia is caused by mechanical and irritant action of nematodes belonging to

282 the order of Strongylida. The biggest one, Dictyocaulus filaria, affects caudal and diaphragmatic 283 part of the lungs, and the small worms, belonging to the Protostrongylidae family 284 (Protostrongylus rufescens, Muellerius capillaris, Cystocaulus ocreatus and Neostronglylus 285 linearis), causes injuries in the dorsal and the diaphragmatic part of the lungs. These nematodes 286 induce a response from the host that tries to surround and encapsulate the parasites, generating the typical nodules associated with verminous pneumonia.

X-ray images show alveolar and nodular interstitial patterns that forms a mixed pattern. Usually, lesions are located in the dorsal part of the lungs, opaquer on the damaged areas.

Ultrasonography reveals an echogenic zone surrounded by normal areas in the dorsal part of the lungs. If the areas of verminous pneumonia are small, they are not easy to see through this technique.

Computed Tomography, again, presents a better picture of nodular pneumonic areas located in the dorsal parts of the lung. Usually, small worms move on to the caudal-dorsal lung, making a line over the dorsal lung tissue with diffused borders (Figs. 12a-12b). An increased thickness of the caudal and diaphragmatic areas can be observed in the case of Dictyocaulus

\subsection{Caseous Lymphadenitis}

Caseous Lymphadenitis (CLA) is a disease caused by Corynebacterium pseudotuberculosis that results in the formation of pyogranulomatous lesions affecting mainly the lymph nodes (Fontaine and Baird, 2008). There are two clinical presentations: the external, also

304 known as cutaneous or superficial, and the visceral form. While superficial form affects only 305 lymph nodes, visceral presentation can affect both lymph nodes and the parenchyma of several 306 organs, especially the lungs. Sometimes, these abscesses can compress other structures such as oesophagus or vagus nerve producing different syndromes (Fuzës et al., 2015).

When CLA affects a mediastinal lymph node, a chest-x-ray displays a radiopaque round 309 mass usually localized forward of the heart, although sometimes, this mass appears behind it.

310 Similar image is observed when the lesion is located in lung parenchyma, kidneys or mesenteric 311 lymph nodes. 
313 abscesses could be found deep inside the parenchyma and transducer cannot provide a clear

314 picture of the CLA. Nevertheless, if abscesses are detected, a hyperechogenic round shape under

315 the normal tissue is shown.

316 Computed tomography provides a specific image of the abscess, their location, and

317 injured tissues involved in the disease. Frequently, there is an enhance area around the abscess

318 and mineralization within abscess due to caseous necrosis (Figs. 13a-13b-13c).

\subsection{Gangrenous pneumonia}

Gangrenous pneumonia, also known as aspiration pneumonia or necrotizing pneumonia, is a pulmonary infection characterized by inflammation and necrosis due to inhalation of foreign substances. Foreign body drives environmental bacteria that produce foci of pulmonary necrosis with accumulation of a foul-smelling exudate that sometimes is also present in the main bronchus and trachea. In these cases, bad smell of exhaled air is a clear clinical sign of the disease (Lacasta et al., 2018).

An X-ray will show a mixed pattern (alveolar and interstitial) where the lesion is located. Affected areas show loss of opacity that sometimes can be mistaken for emphysema, although usually the lesion is surrounded by clear border.

Ultrasound images are clear, offering different echogenicity foci in all the affected area depending on the material inside the lesion (Fig. 14a).

Computed tomography shows the lesion with the adjacent tissues enhanced. Affected area shows necrotic tissue (dark or black) with diffused edges (Fig. 14b). Bronchia lumen cannot

\section{Final remarks}

Diagnostic imaging techniques are very useful tools for the proper diagnosis of respiratory diseases in sheep. The use of ultrasound and thermographic cameras must be enhanced

342 at farm level, not only with reproductive purpose, but also with the objective of assisting in the diagnosis of major diseases present in sheep farms.

X-ray and, particularly, computed axial tomography are very interesting tools to facilitate

345 the understanding of the main pathological processes in sheep, especially at the respiratory level,

346 as it has been shown in this article. CT images allow analyzing slides of tissues of different 
347 thicknesses that offer very detailed images. These pictures may sometimes even improve the

348 diagnostic results obtained at the post mortem study of the animals, because the structure of the

349 tissues can be observed in greater detail. Furthermore, these techniques let us to observe the

350 evolution of the lesions and how animals are recovering.

\section{5. Acknowledgment} scan. Furthermore, we would like to acknowledge Equine service veterinarians of the Veterinary Hospital at the University of Zaragoza for helping us with the x-ray interpretation.

\section{Conflict of interest statement}

The authors have nothing to disclose.

\section{References}

Alcaide, M., Reina, D., Sanchez, J., Frontera, E., Navarrete, I. (2003). Seasonal variations in the larval burden distribution of Oestrus ovis in sheep in the southwest of Spain. Veterinary Parasitology 118, 235-241.

Benavides, J., Gonzalez, L., Dagleish, M., Perez, V. (2015). Diagnostic pathology in microbial diseases of sheep or goats. Veterinary Microbiology 181, 15-26.

Cousens C., Thonur L., Imlach S., Crawford J., Sales J. et al. (2009) Jaagsiekte sheep retrovirus is present at high concentration in lung fluid produced by ovine pulmonary adenocarcinoma-affected sheep and can survive for several weeks at ambient temperatures. Research in Veterinary Science, 87, 154-156.

De las Heras, M., Ortin, A., Cousens, C., Minguijon, E., Sharp, J.M. (2003). Enzootic nasal adenocarcinoma of sheep and goats, In: Fan, H. (Ed.) Jaagsiekte Sheep Retrovirus and Lung Cancer. Current Topics in Microbiology and Immunology 275, 201-223.

De las Heras, M., Ortín, A., Borobia, M., Navarro, T. (2018). Enzootic Nasal Adenocarcinoma: an update. Small Ruminant Research (2018)

Ferrer, L.M., Garcia de Jalon, J.A., De las Heras, M. (2002). Athlas of ovine pathology. Servet Diseño y Comunicacion S.L. 184-187.

Fontaine, M.C., Baird, G.J. (2008). Caseous lymphadenitis. Small Ruminant Research 76, 42-48.

Füzes, K., Osorio, V., Lacasta, D. (2015). Timpanismo de rumen y abomaso en una oveja causado por una linfoadenitis caseosa. Número especial Septiembre 2015. Monográficos Albeitar.

Gracia, M. J., Lucientes, J., Peribanez, M. A., Castillo, J. A., Calvete, C., Ferrer, L. M. (2010). Epidemiology of Oestrus ovis infection of sheep in northeast Spain (mid-Ebro Valley). Tropical Animal Health and Production 42(5), 811-813.

Gracia, M.J., Ruíz de Arcaute, M., Ferrer, L.M.; Ramo, M., Jimenez, C., Figueras, L. Oestrosis: parasitism by Oestrus ovis (2018) 
Lacasta, D., Ferrer, L.M., Ramos, J.J., Bueso, J.P., Borobia, M., de Arcaute, M.R., Figueras, L., Gonzalez, J.M., De las Heras, M. (2012). Chronic proliferative rhinitis associated with Salmonella enterica subspecies diarizonae in sheep in spain. Journal of Comparative Pathology 146, 72-72.

\section{Figure legends}

430 Figure 1. 1a. Healthy animal. 1b. Thermogram of a healthy animal with normal refrigeration of 431 the nostril (White circle). 1c. ENA affected animal (black arrow). 1d. Thermogram of an ENA

432 affected animal. Reddish or white colours in the posterior segment of the nose matching with the 

obstruction and poor ventilation of the area (white circle).

Figure 2. 2a. Lateral radiographic projection of the head of a sheep with ENA (white arrow). $2 b$. Ultrasound image of the ethmoidal turbinate in a case of advance enzootic nasal adenocarcinoma in sheep. 2c. CT axial view of a ewe with enzootic nasal adenocarcinoma (bone filter), showing the affection of the nasal bone. $2 \mathrm{~d}$. CT 3D view of a sheep with enzootic nasal tumour (volume rendering). The lithic process in the nasal and lacrimal bones producing some holes in the bones is shown (white arrows).

Figure 3. 3a. Thermogram of a ewe with unilateral chronic proliferative rhinitis. White areas indicate swollen turbinate and the inability of a correct nasal cavity ventilation. 3b. Lateral radiographic projection of a ewe with chronic proliferative rhinitis affecting the ventral nasal turbinate. Swollen area is clearly seen in the image. 3c. CT axial image of a ewe with chronic proliferative rhinitis (bone filter). It can be observed the tissue invasion from the right to the left nasal chamber causing the nasal septum deformation (white arrow). 3d. CT 3D image of a ewe with chronic proliferative rhinitis (volume rendering).

Figure 4. 4a. Lateral radiographic projection of the head of a ewe parasitized by Oestrus ovis larvae. It is evident the difficulty to distinguish the larvae from the other tissues (white arrow). 4b. CT axial view of the head of a ewe parasitized by Oestrus ovis larvae (head filter). Larvae are shown in the picture (white arrow). 4c. CT sagittal view of the head of a ewe parasitized by

457 Figure 5. 5a. Ventrodorsal radiographic projection of the head of a ewe with a nasal abscess 458 caused by Corynebacterium pseudotuberculosis. 5b. Thermogram of a ewe with an abscess and 459 rhinitis in the right side. $5 \mathrm{c}$. CT of the head of a ewe with a nasal abscess (bone filter). Destruction 460 of the nasal bone is cleared shown (white arrow). 5d. CT coronal view (soft tissue filter) of the 461 abscess injuring the right nasal chamber and the right nasal bone of a ewe (white arrows). (Soft 462 tissue filter).

464 Figure 6. 6a. CT sagittal view of a ewe's head with an abscess (soft tissue filter). 6b. CT 3D axial 465 view of a ewe's head with an abscess (volume rendering). A hole (white arrow) in the nasal and maxillary bone caused by an abscess is shown (yellow circle). 
468 Figure 7. 7a. CT sagittal view of a ewe's head with an abscess (soft tissue filter). 7b. CT 3D axial 469 view of a ewe's head with an abscess (volume rendering). A hole (white arrow) in the nasal and 470 maxillary bone caused by an abscess is shown (yellow circle). 7c. Ultrasonographic image of the 471 neck of a ewe with a $7.27 \mathrm{~cm}$ abscess of caseous lymphadenitis pressing and deforming the 472 laryngx. 7d. CT axial view of the neck of a ewe with a caseous lymphadenitis pyogranuloma 473 pressing the larynx (soft tissue filter).

475 Figure 8. 8a. Lateral radiographic projection of the neck of a ewe with tracheal crushing. It is 476 showed the stenosis in the medial fraction of the trachea. 8b. CT 3D sagittal view of the trachea 477 of a sheep with the crushing of some tracheal rings.

479 Figure 9. 9a. Lateral radiographic projection of the thorax of a sheep with interstitial pattern.

480 There is an increased opacity in the lungs due to infiltrate of lymphocytes in the interstitial tissue.

$4819 \mathrm{~b}$. Ultrasound imaging shows consolidated lung with high echogenicity in a clinical case of 482 Maedi Visna disease. 9c. CT image of the thorax of a sheep with high opacity caused by interstitial 483 pneumonia associated with Maedi Visna disease.

Figure 10. 10a. Radiography of the thorax of a sheep affected of ovine respiratory complex. It can be observed the alveolar pattern, expressed as white areas and diffused borders. 10b. Ultrasonographic imaging of a lung with fibrinous pneumonia associated with ovine respiratory complex. More echogenic tissue can be clearly observed. 10c. CT image of the thorax of a sheep

Figure 11. 11a. Thorax radiography of a ewe with ovine pulmonary adenocarcinoma. Nodular pattern with different-sized nodules is observed. 11b. Thorax ultrasonography of a ewe with ovine 494 pulmonary adenocarcinoma. Echogenic nodules with different sizes and shapes are shown. 11c. 495 CT axial view of the thorax of a ewe with ovine pulmonary adenocarcinoma (soft tissue filter). 496 Ventral lobes with consolidated areas and metastatic nodules in the rest of the lung are shown.

498 Figure 12. 12a. CT sagittal view of the thorax of a ewe with two verminous pneumonic areas in 499 the dorsal left lung (white arrows). 12b. CT 3D sagittal view of the lung of a ewe with verminous 500 pneumonia (volume rendering). Four foci are indicated in the dorsal lobe (white arrows).

502 Figure 13. 13a. CT sagittal view of the thorax of a ewe with caseous lymphadenitis in the 
503 mediastinal lymph node (soft tissue filter) (white arrow). 13b. CT 3D image of caseous

504 lymphadenitis affecting mediastinal lymph node (volume rendering) (white arrow). 13c. CT 3D

505 image of caseous lymphadenitis (volume rendering) affecting mediastinal lymph node (white

506 arrow).

508 Figure 14. 14a. Ultrasound image of the lung of a sheep affected of gangrenous pneumonia.

509 Different echogenicity foci can be observed. 14b. CT sagittal view of the thorax of a ewe with

510 gangrenous pneumonia (soft tissue filter). White arrow indicates the more severe affected areas

511 with necrotic tissue within them. 14c. CT axial view of a gangrenous pneumonia in sheep (soft

512 tissue filter) that show a huge necrotic area in the right lung (white arrow).

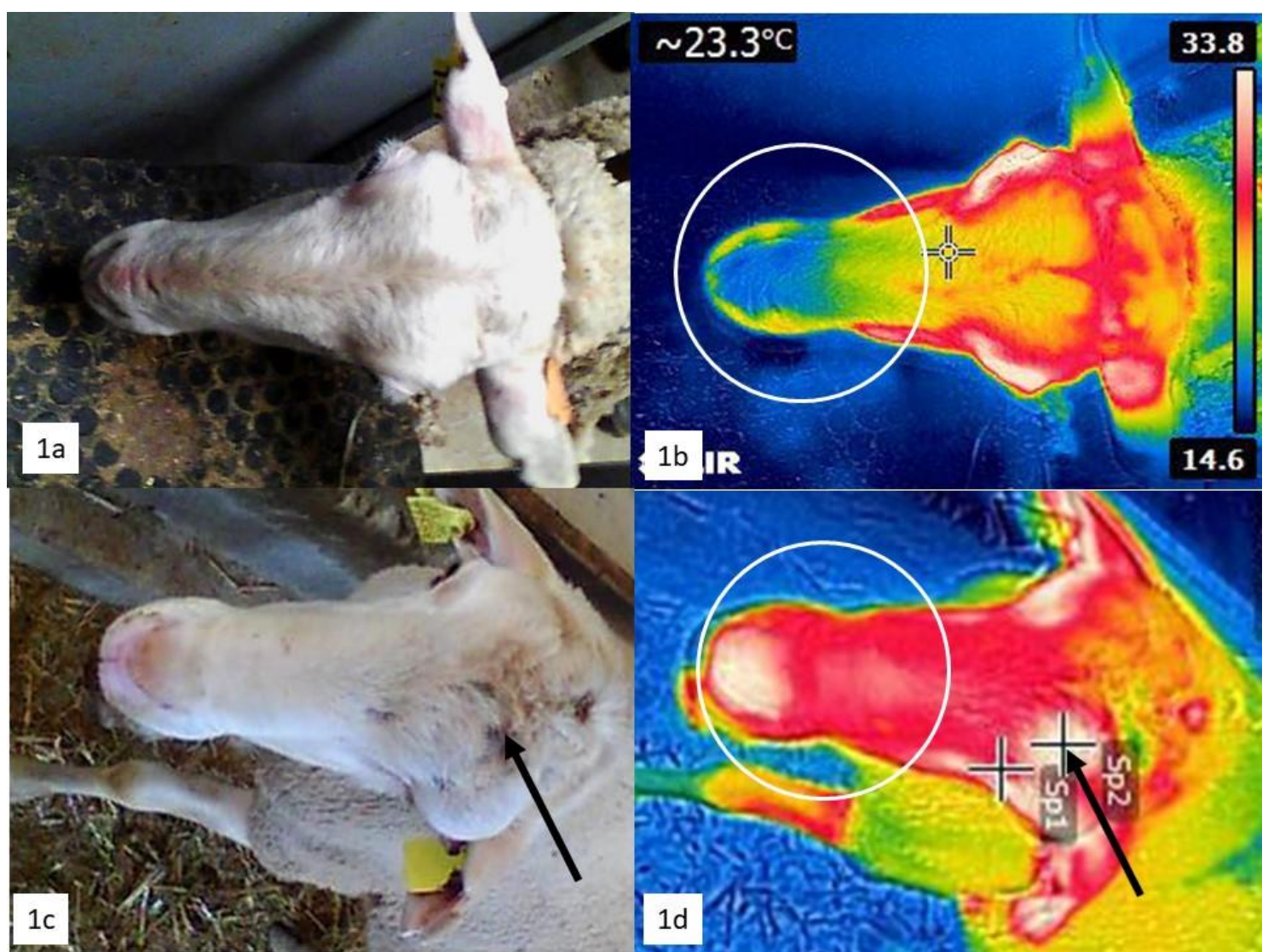



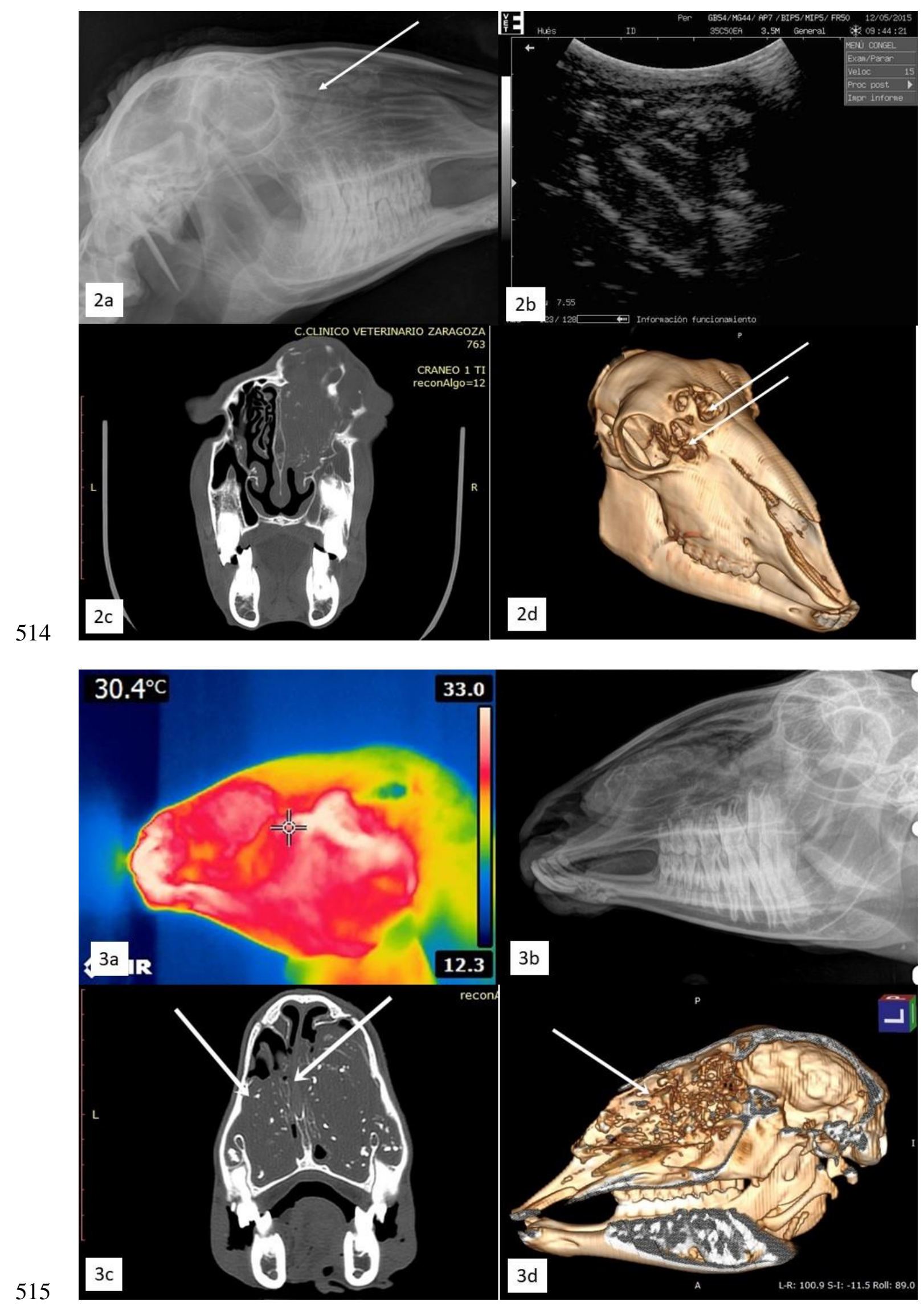

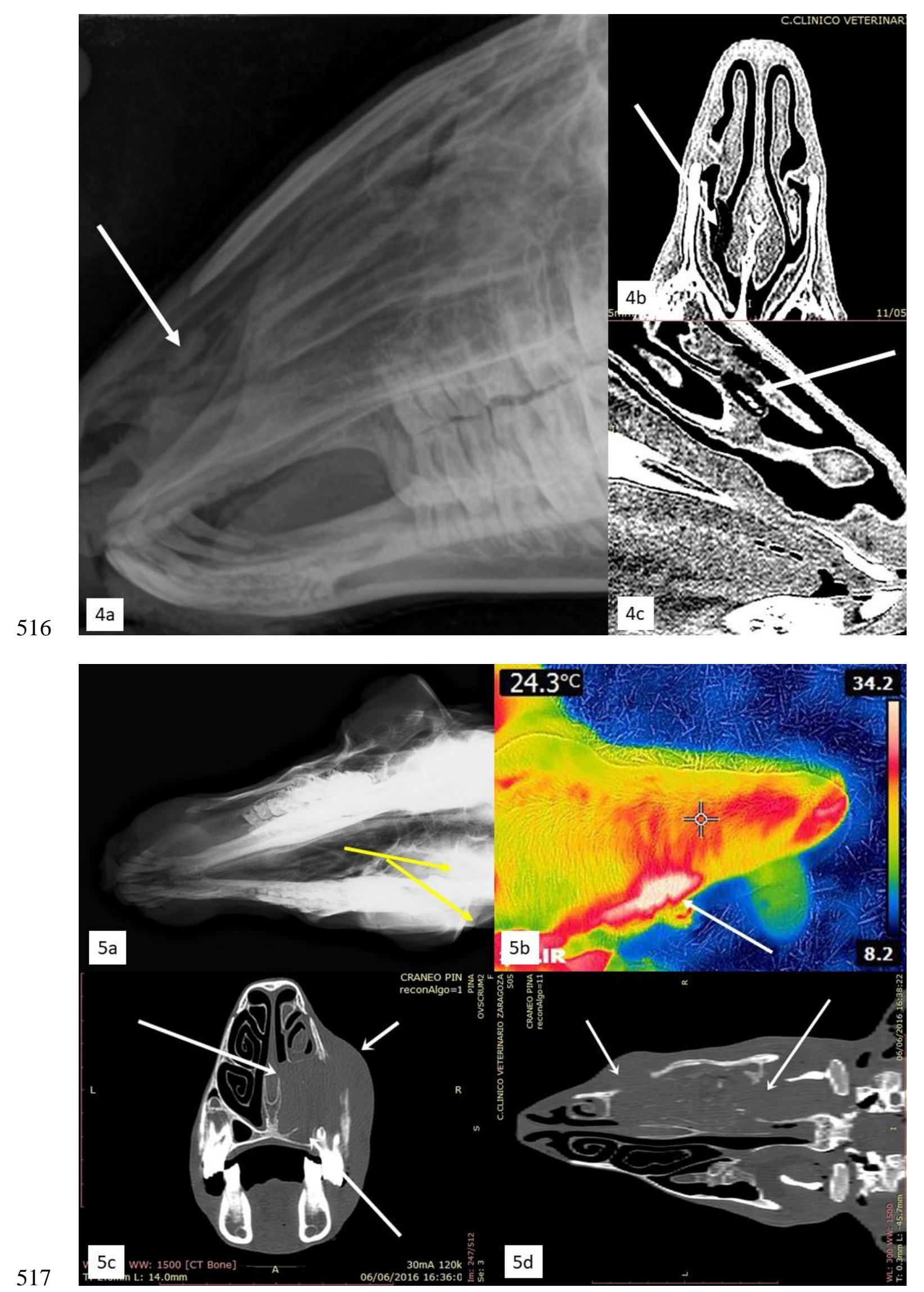

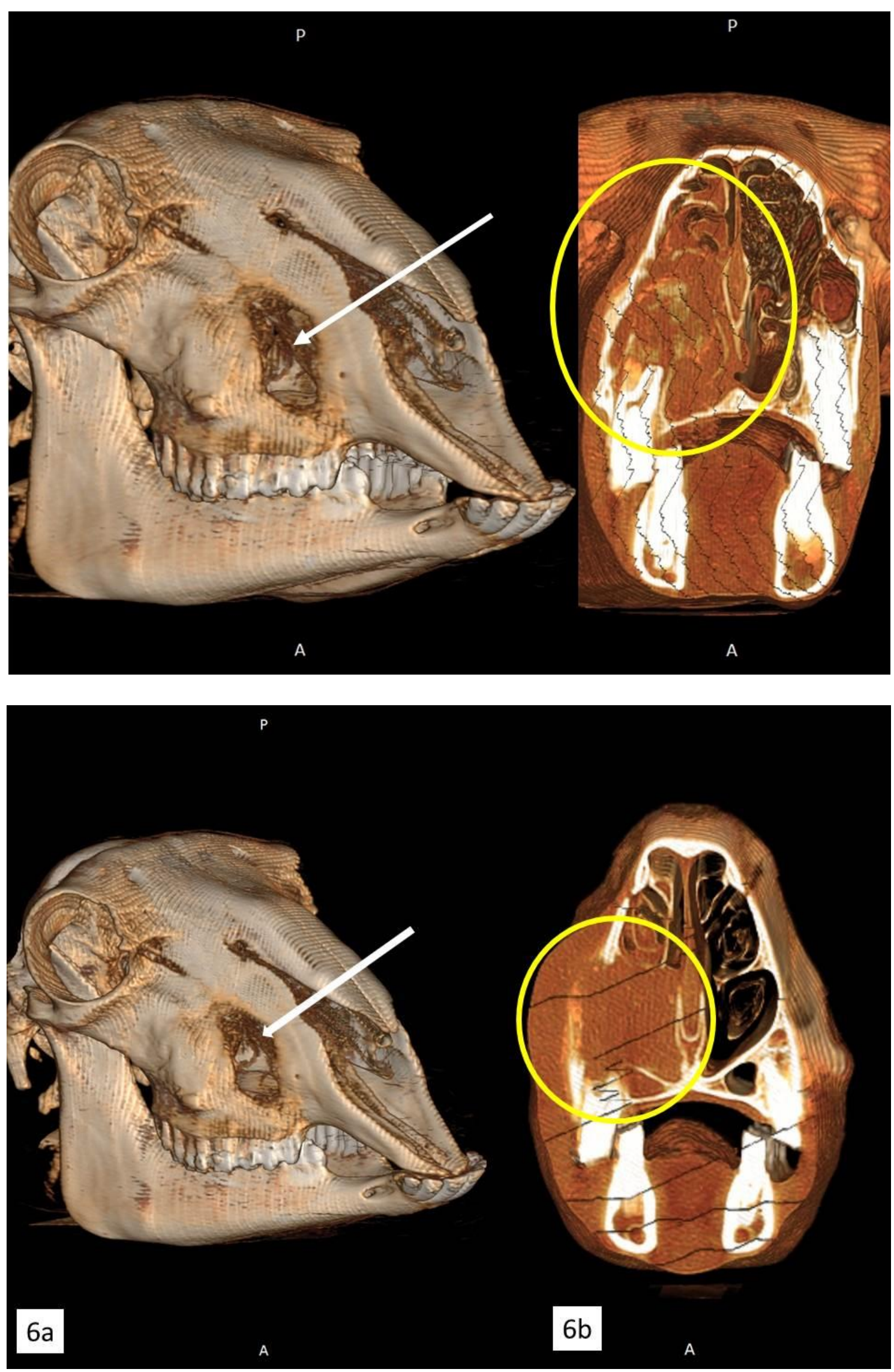

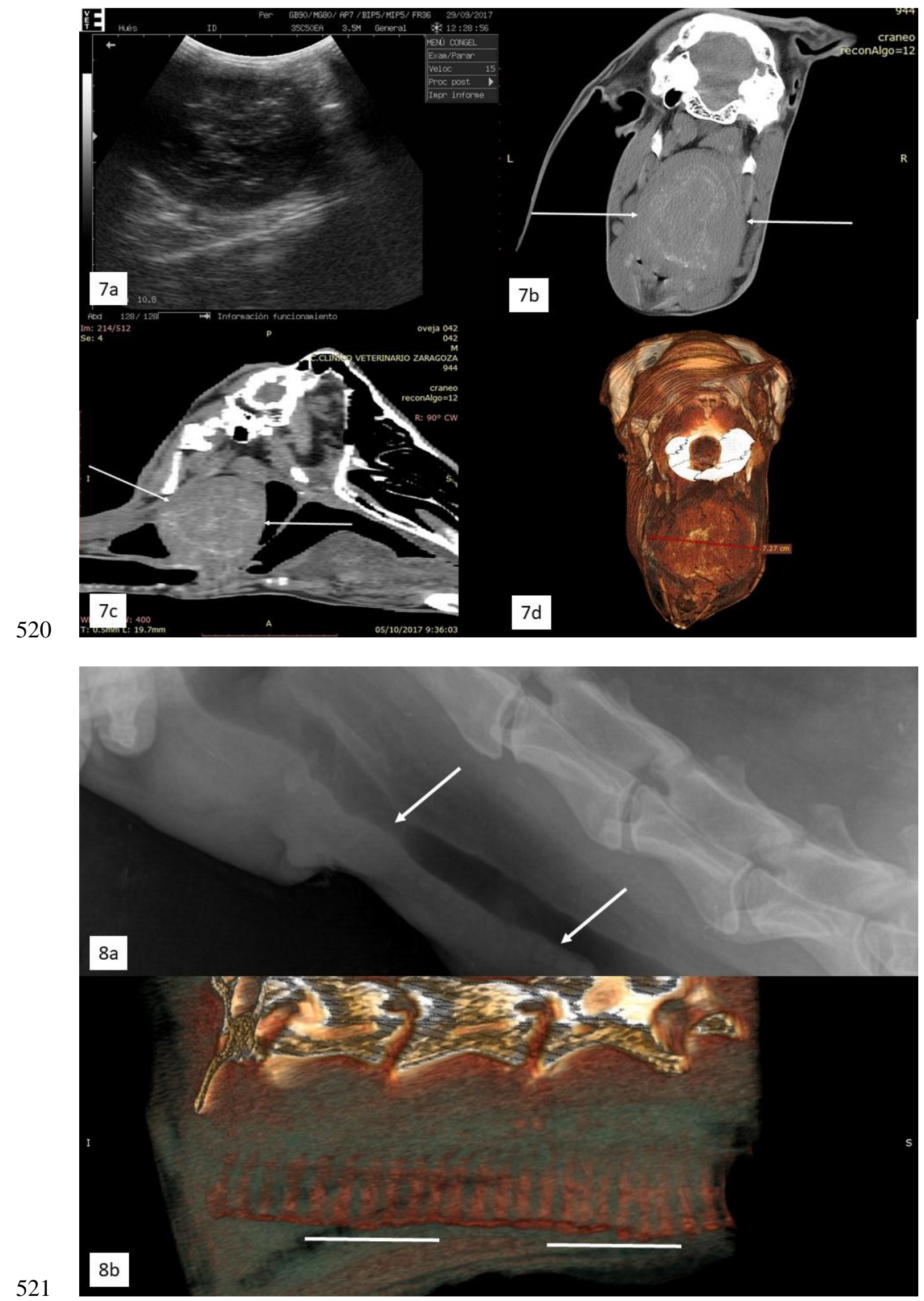

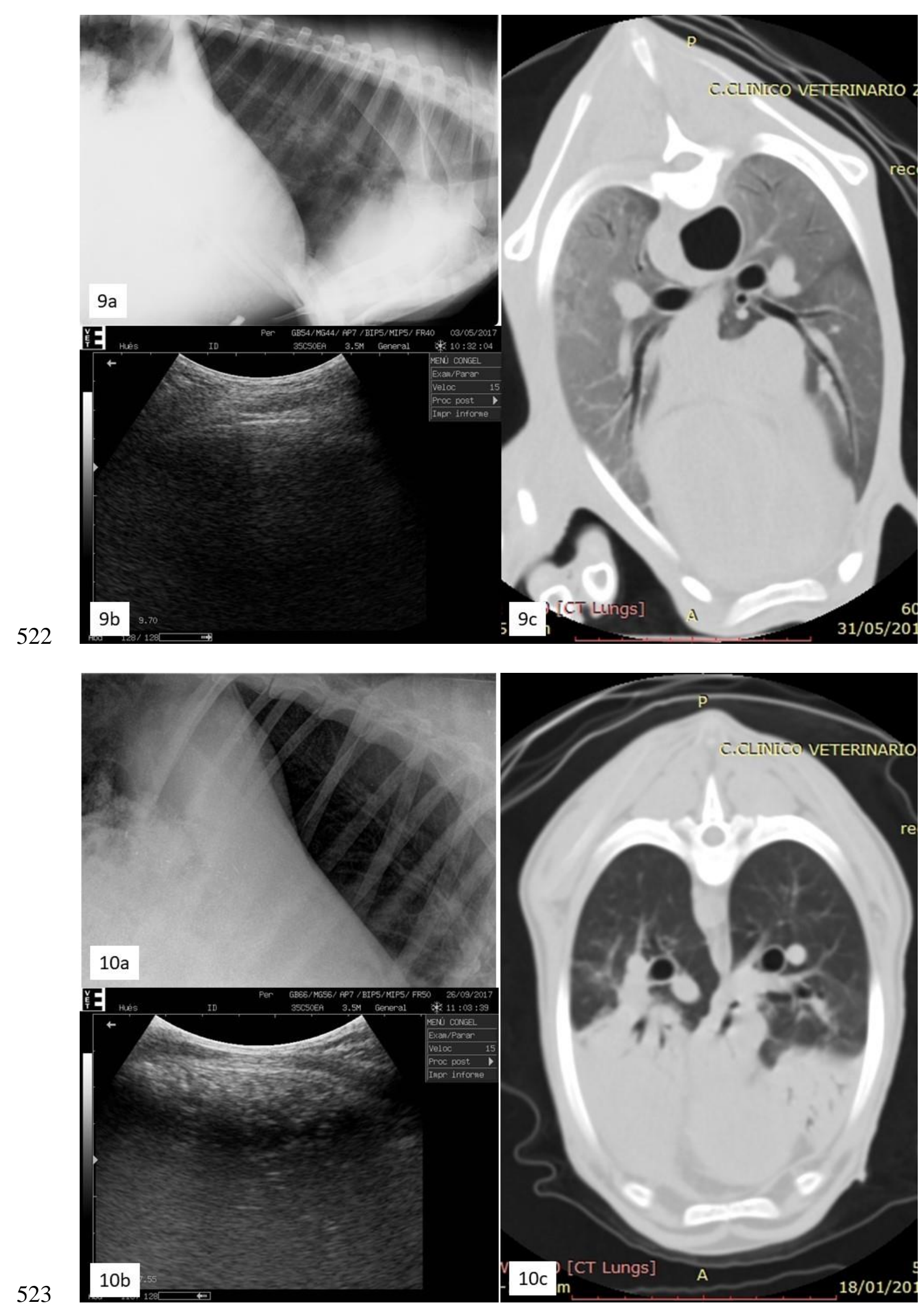

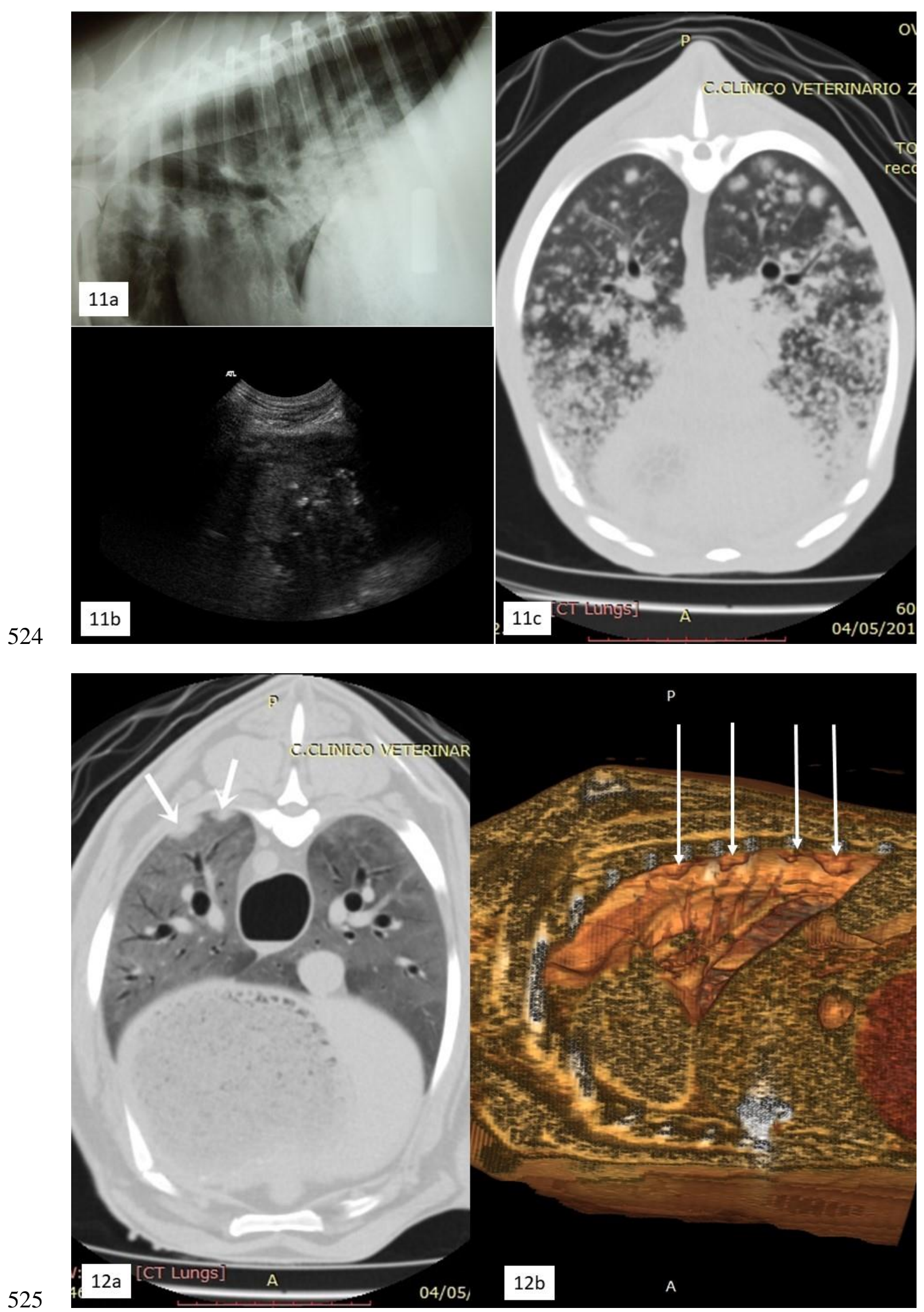

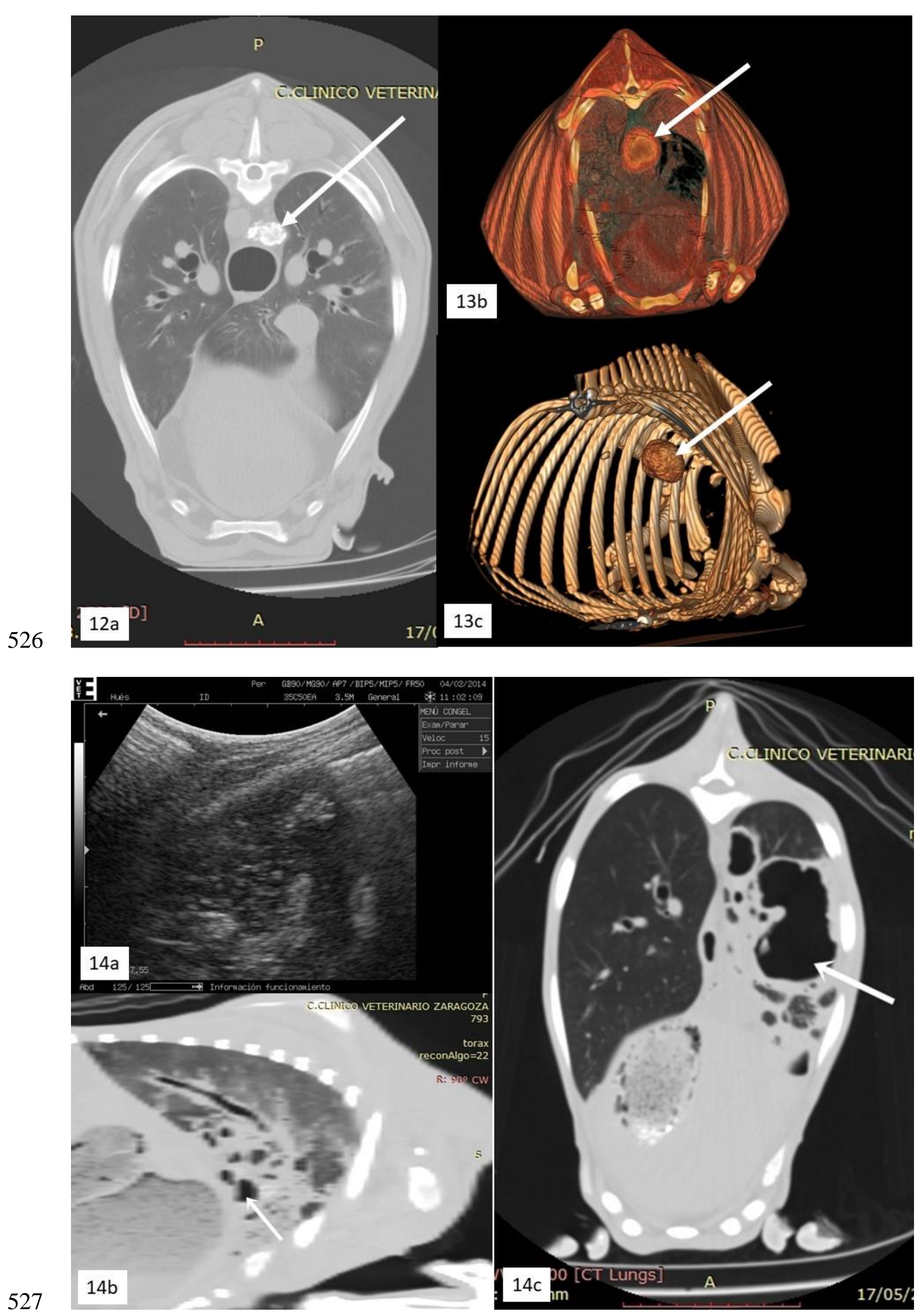